\title{
Surface resistivity estimation by scanning surface potential microscopy
}

\author{
Z. Rakocevic, ${ }^{1, a)}$ N. Popovic, ${ }^{1}$ Z. Bogdanov, ${ }^{1}$ B. Goncic, ${ }^{1}$ and S. Strbac ${ }^{2}$ \\ ${ }^{1}$ Laboratory of Atomic Physics, INS Vinca, P.O. Box. 522, 11001 Belgrade, Serbia \\ ${ }^{2}$ ICTM-Institute of Electrochemistry, University of Belgrade, P.O. Box 815, 11001 Belgrade, Serbia
}

(Received 19 February 2008; accepted 6 May 2008; published online 3 June 2008)

\begin{abstract}
Nickel was sputter deposited on a glass with a thin film thickness of $600 \mathrm{~nm}$ under either in an argon atmosphere or under a partial pressure of nitrogen of either $1.3 \times 10^{-4}$ or $4 \times 10^{-4}$ mbar. Atomic force microscopy and scanning surface potential microscopy (SSPM) were used to study the morphology and to estimate the surface resistivity of the obtained $\mathrm{Ni}$ thin films taking into account surface-roughness effects. For the three samples investigated, the surface resistivity values as estimated using SSPM were in good agreement with the results obtained by standard four-point probe measurements. (0) 2008 American Institute of Physics. [DOI: 10.1063/1.2937647]
\end{abstract}

With the advent of secondary imaging modes derived from tapping mode atomic force microscope (AFM), the assessment of local electrical properties of thin films provided by the mapping of the local surface potential distribution, is enabled. ${ }^{1,2}$ In this work, for the first time, an estimation of the local resistivity of thin films was derived from the local surface potential distributions. Nickel thin films sputter deposited on glass were examined. With increasing partial pressure of nitrogen, the grain size, and consequently, the surface roughness increased, which might be induced by different growth mechanisms of Ni films and new phases formation as revealed in earlier studies. ${ }^{3-5}$

The deposition of nickel onto a polished glass substrate was performed in a Bulzers Sputtron II vacuum system under an argon atmosphere or under a partial pressure of nitrogen of $1.3 \times 10^{-4}$ or $4 \times 10^{-4}$ mbar. The residual pressure in the chamber was $7 \times 10^{-6} \mathrm{mbar}$ and the partial pressure of argon was $1.3 \times 10^{-3}$ mbar. All depositions were performed at a substrate temperature of $100{ }^{\circ} \mathrm{C}$. The thickness of the deposited nickel films was measured using a profilometer Talystep I, with an error less than 5\%. A scanning probe microscope (SPM) Quadrex Multimode IIIe (Veeco Instruments), operated under ambient conditions, was used in this work. The topography was acquired in the main scan employing the standard tapping mode of the AFM using a commercial Veeco MESP tip. The tip was subsequently retraced in lift mode with a constant tip-sample distance (lift scan height) of $20 \mathrm{~nm}$. By varying the voltage of the tip, the potential difference between the tip and the sample is nullified so that the tip bias is a direct measure of surface potential. Both the root mean square (rms) surface roughness and surface potential were estimated using the subprogram packages of the SPM, as the average value out of images taken from different points within the substrate. The sheet resistance of the films were measured by a four-point probe technique at room temperature. The resistivity values, $\rho$, were deduced from the sheet resistance $R / \square$, and film thickness.

Here, surface-roughness effects on the electrical resistivity of thin films will be considered. Based on a comprehen-

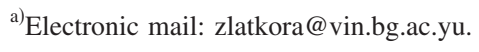

sive theoretical work related to the evaluation of threedimensional (3D) surface parameters, various surface functions valid for two-dimensional surface topography ${ }^{6,7}$ can be applied to the complete 3D dataset using simple mathematical extensions. ${ }^{8}$ Thus, the surface roughness is characterized by the roughness exponent $\alpha(0 \leqslant \alpha \leqslant 1)$, the in plane correlation area $S_{0}$ and the rms roughness amplitude $H$. The rms roughness amplitude, $H \propto S^{2 \alpha}$, is a function of the area over which it is measured until the roughness saturates at some critical value $S_{0}$. Plotting $H$ versus $S$ on a $\log -\log$ scale gives a straight line with a slope $\alpha$. Comprehensive studies of surface-roughness effects on the conductivity of thin films have shown that the surface conductivity, $\sigma$, is proportional to $\left(S_{0} \alpha\right) / H,{ }^{9}$ not taking into account grain boundary scattering effects. Analogously to the estimation of the rms surface roughness from AFM measurements, the rms surface potential $E$ can be estimated from scanning surface potential microscopy (SSPM) measurements. Since SSPM images are a direct measure of the potential distribution over the surface, they involve grain boundary effects. The corresponding in plane correlation area $S_{0 E}$ and the surface potential exponent $\alpha_{E}$ can be calculated as well. Therefore, the conductivity as being directly related to the surface potential, $E,{ }^{10}$ can be expressed as being proportional to $E /\left(S_{0 E} \alpha_{E}\right)$. This gives the following expression for the surface resistivity $\left(\rho=\sigma^{-1}\right)$ :

$$
\rho \approx \frac{H}{E} \frac{S_{0 E} \alpha_{E}}{S_{0} \alpha},
$$

involving grain boundary effects at the temperature of measurements.

AFM images showing the morphology of $\mathrm{Ni}$ thin films obtained under various deposition conditions and the corresponding SP images, showing the surface potential distribution are presented in Fig. 1. The AFM images show that the $\mathrm{Ni}$ thin films in all cases were characterized by a grained structure. Section analysis of the AFM images showed that the mean grain size for the $\mathrm{Ni}$ thin film obtained under an argon atmosphere was about $60 \mathrm{~nm}$ [Fig. 1(a)]. Compared to this, a finer grained structure $(35 \mathrm{~nm})$ was observed for the $\mathrm{Ni}$ thin film obtained under a partial nitrogen pressure of $1.3 \times 10^{-4}$ mbar [Fig. 1(b)], while a larger grained structure 

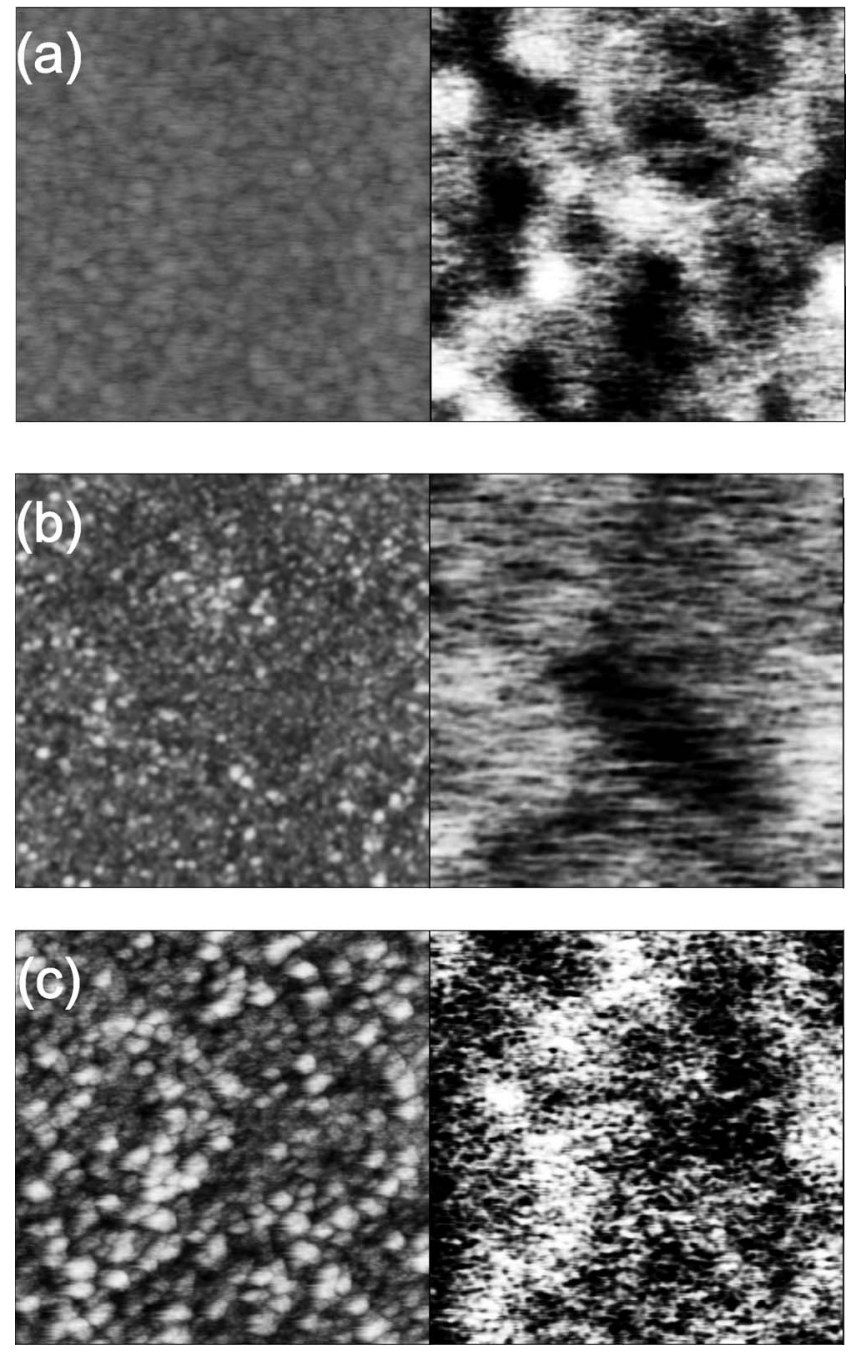

FIG. 1. (a) AFM images (left) and corresponding SP images (right), $\left(2 \times 2 \mu \mathrm{m}^{2}\right)$, of $600 \mathrm{~nm}$ thick $\mathrm{Ni}$ thin film deposited on a glass: (a) under an argon atmosphere; (b) under a partial nitrogen pressure of $1.3 \times 10^{-4} \mathrm{mbar}$; (c) under a partial nitrogen pressure of $4 \times 10^{-4}$ mbar.

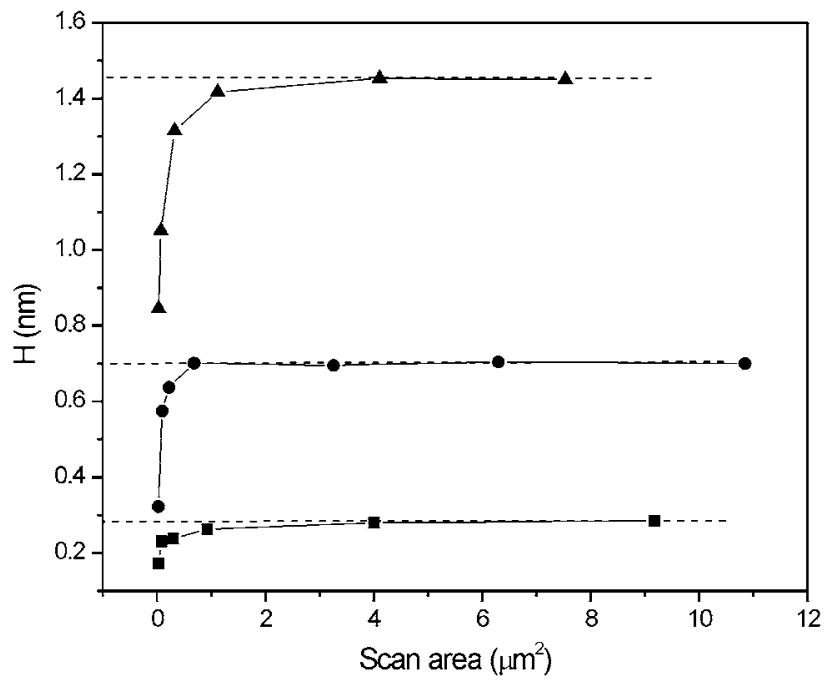

FIG. 2. The dependence of the rms surface roughness on the scan area for the samples: (a) (-口-), (b) (--), and (c) (- $\mathbf{\Delta}-$-). (See Fig. 1 for the deposition conditions.)

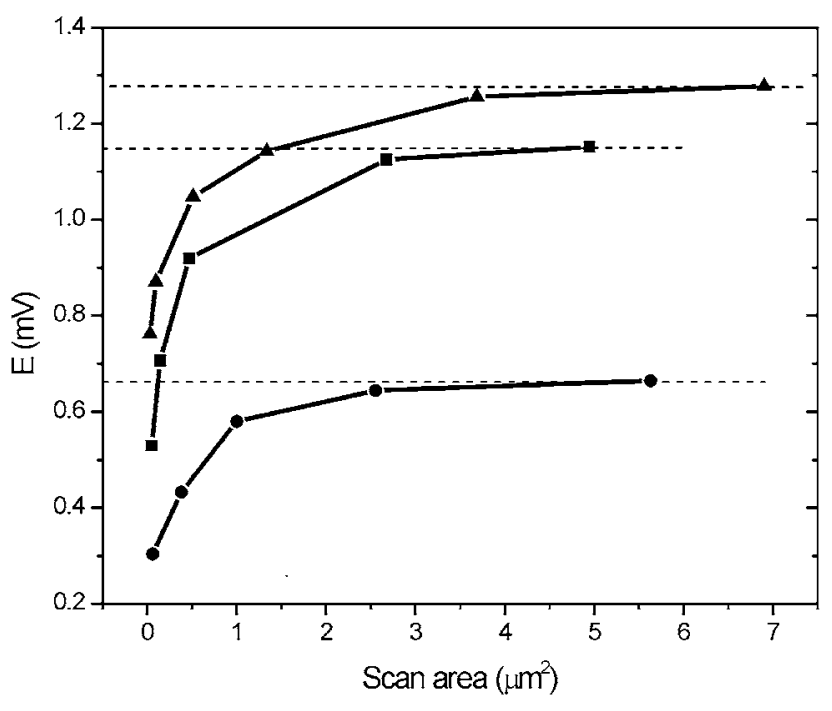

FIG. 3. The dependence of the average surface potential on the scan area for

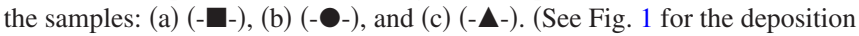
conditions.)

with a mean grain size of $65 \mathrm{~nm}$, was observed for the film obtained under a partial nitrogen pressure of $4 \times 10^{-4} \mathrm{mbar}$ [Fig. 1(c)]. The changes in the grain size with increasing partial pressure of nitrogen confirm the influence of nitrogen on the refinement of the $\mathrm{Ni}$ grains, with the enlargement of the grains most likely being due to the formation of a new phase. The corresponding SSPM images revealed a heterogeneous surface potential distribution, with a pronounced surface potential difference for the larger grained films. It can be seen that adjacent grains had a very similar surface potential, thus forming domains of high and low surface potentials (bright and dark areas on the images). Therefore, both the average surface roughness and the average surface potential were calculated for various scan areas.

The dependences of the average surface roughness on the scan area for samples from Figs. 1(a)-1(c), are presented in Fig. 2. The sample from Fig. 1(a) exhibited the lowest,

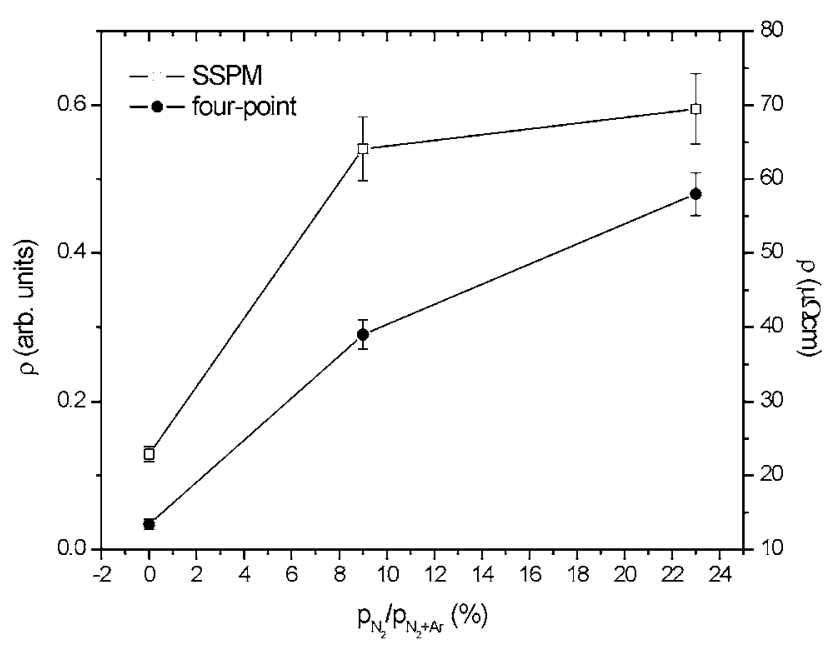

FIG. 4. Average surface resistance measured using SSPM (- $\square-$ ), (the left $y$ axis) and by the four-point method (--), (the right $y$ axis). The samples investigated can be identified by the partial nitrogen pressure during preparation, given on the $x$ axis. 
while the sample from Fig. 1(c) exhibited the highest surface roughness, irrespective of the average grain size. The in plane correlation area $S_{0}\left(0.13 \pm 0.02 \mu \mathrm{m}^{2}\right)$ calculated from Fig. 2, as well as the roughness exponent $\alpha(0.46 \pm 0.06)$ calculated plotting $H-S$ on a log-log scale are practically the same for all samples.

The dependences of the average surface potential on the scan area for the same samples are presented in Fig. 3. Contrary to the previous case, the lowest average surface potential corresponds to the sample with the smallest grain size [Fig. 1(a)], and increases with increasing grain size (Figs. 1 and 3). The in plane correlation area $S_{0 E}$ is $0.78 \pm 0.09 \mu \mathrm{m}^{2}$, while the potential exponent $\alpha_{E}$ is $(0.04 \pm 0.01)$, similar for all three samples. It should be noted that the value $S_{0 E}$ represents the smallest area for which an estimation of the surface resistivity makes sense.

Since, according to the above consideration, the surface resistivity of the samples is related to both the surface roughness $H$ and surface potential $E$, it was calculated using Eq. (1), and the results presented in Fig. 4 in arbitrary units for the three investigated samples. The values obtained are in accordance with the values obtained using standard four- point probe measurements. We demonstrate in this work that quality measurements using surface potential imaging are useful for comparison of the surface resistivity of different samples.

We gratefully acknowledge the Ministry of Science of Republic Serbia for financial support of the Project No. 06-141001.

${ }^{1}$ S. Habouti, C. Solterbeck, and M. Es-Souni, Appl. Phys. Lett. 88, 262903 (2006).

${ }^{2}$ Y. Miyato, K. Kobayashi, K. Matsushige, and H. Yamada, Nanotechnology 18, 084008 (2007).

${ }^{3}$ N. Popovic, T. Dimitrijevic, Z. Bogdanov, B. Goncic, S. Strbac, and Z. Rakocevic, Vacuum 48, 705 (1997)

${ }^{4}$ N. Popovic, Z. Bogdanov, B. Goncic, S. Zec, and Z. Rakocevic, Thin Solid Films 343, 75 (1999).

${ }^{5}$ D. Vempaire, S. Mraglia, A. Sulpice, L. Ortega, E. K. Hlil, D. Fruchart, and J. Pelletier,J. Magn. Magn. Mater. e843, 272 (2004).

${ }^{6}$ F. Family, Physica A 168, 561 (1990).

${ }^{7}$ W. M. Tong and R. S. Williams, Annu. Rev. Phys. Chem. 45, 401 (1994).

${ }^{8}$ W. P. Dong, P. J. Sullivan, and K. J. Stout, Wear 178, 29 (1994).

${ }^{9}$ G. Palasantzas and J. Barnas, Phys. Rev. B 56, 7726 (1997).

${ }^{10}$ Z. Hashin and S. Shtrikman, Phys. Rev. 130, 129 (1963). 